\title{
МОДЕЛИРОВАНИЕ ПОВЕРХНОСТИ ЛИСТ МЕБИУСА
}

\section{MODELING OF A MOBIUS SHEET}

\section{Vavanov}

A. Stepura

Summary. The article discusses two ways of modeling a topological surface - a Mobius sheet in the Wolfram Mathematica program and in the 3DMax graphics editor. Several variants of visual representation of the surface are presented. Recently, architects and designers have shown interest in creating art objects and structures using this surface. This is due to the new possibilities that computer modeling provides in the study of surface properties by using electronic and information models of similar objects. The Mobius Sheet surface considered in the article is very promising in terms of a new look at its geometric properties revealed in the process of information modeling.

Keywords: Mobius sheet, information modeling technologies (BIM), Wolfram Mathematica, 3DMax graphic editor.

и нтерес к нетривиальным поверхностям в архитектуре и дизайне проявился в полной мере в конце XX века. Популярность творений Нормана Фостера и Захри Хадид обусловлена применением в геометрической схеме и оболочках зданий [1] топологических поверхностей (рис. 1). Развитие технологий информационного моделирования (BIM) ускоряет процесс проектирования и строительства сложных поверхностей. Для внедрения таких подходов в практику проектирования необходимо разрабатывать алгоритмы моделирования различных поверхностей в графических редакторах.

Лист Мебиуса - это односторонняя поверхность [2, с. 47], описываемая либо параметрически, либо неявным уравнением третьего порядка. Кинематический эту поверхность можно получить таким образом: в качестве образующей надо взять отрезок, в качестве направляющей - любую замкнутую плоскую кривую (например, окружность), и в процессе движения одновременно вращать отрезок относительно его средней точки на угол 180 градусов за полный оборот вдоль направляющей.

В параметрической форме уравнение этой поверхности представляется следующим образом [3]:

$$
x(t, \varphi)=\left(a+\sin \frac{\varphi}{2}\right) \cos \varphi
$$

Ваванов Дмитрий Алексеевич

Преподаватель, Московский государственный строительный университет

Степура Анна Вячеславовна

Преподаватель, Московский государственный строительный университет stepura83@mail.ru

Аннотация. В статье рассмотрены два способа моделирования топологической поверхности - Лист Мебиуса в программе Wolfram Mathematica и в графическом редакторе $3 \mathrm{dMAX}$. Представлены несколько вариантов наглядного изображения поверхности. В последнее время, со стороны архитекторов и дизайнеров обозначился интерес к созданию арт-объектов и сооружений с использованием указанной поверхности. Это связано с теми новыми возможностями, которые дает компьютерное моделирование в исследовании свойств поверхностей путем использования электронных и информационных моделей подобных объектов. Рассматриваемая в статье поверхность Лист Мебиуса весьма перспективна в плане нового взгляда на ее геометрические свойства, выявляющиеся в процессе информационного моделирования.

Ключевые слова: лист Мебиуса, технологии информационного моделирования (BIM), программе Wolfram Mathematica, графический редактор 3dMAX.

$$
\begin{aligned}
& y(t, \varphi)=\left(a+t \sin \frac{\varphi}{2}\right) \sin \varphi \\
& z(t, \varphi)=t \cos \frac{\varphi}{2}
\end{aligned}
$$

где, $a$ - радиус направляющей окружности для отрезка, образующего поверхность ленты Мебиуса;

$t$ - параметр, определяющий ширину ленты, $-1<t<1$;

$$
0<\varphi<2 \pi
$$

Изображение, полученное по этим уравнениям в программе Wolfram Mathematica, показано на следующих рисунках (в различных ракурсах) (рис. 2):

При использовании неявной формы задания, уравнение поверхности имеет следующий вид [4]:

$$
\begin{aligned}
& -y * a^{\wedge} 2+y * x^{\wedge} 2+y^{\wedge} 3-2 * a * x * z- \\
& -2 * z * x^{\wedge} 2-2 * z * y^{\wedge} 2+y * z^{\wedge} 2=0
\end{aligned}
$$

И форма поверхности несколько меняется (изображение получено в программе Wolfram Mathematica). Изменение внешнего вида связано с тем, что в этом варианте поверхность Мебиуса представлена не в виде ленты с определенной шириной, а в виде неограничен- 


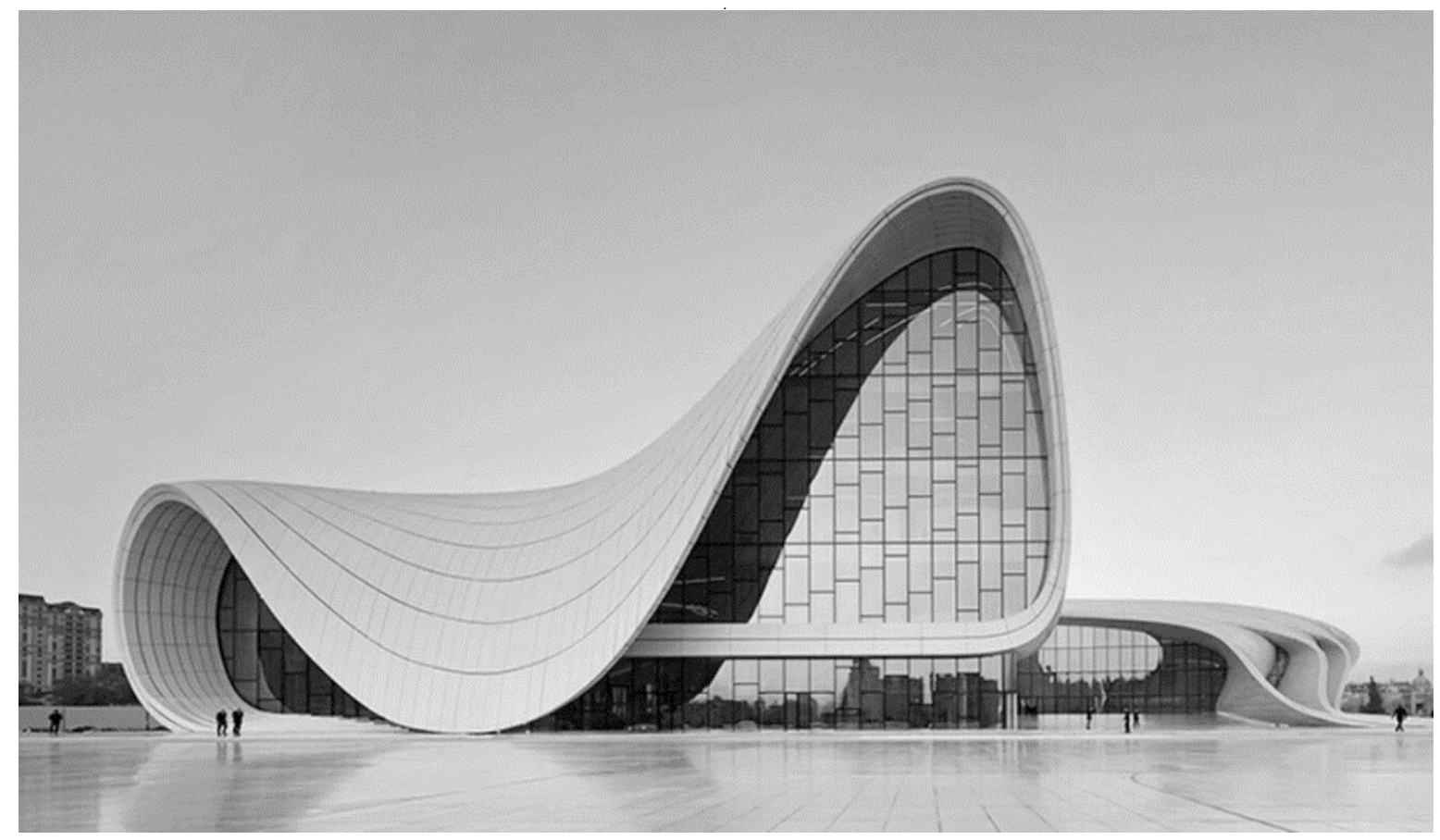

Рис. 1. Центр Гейдара Алиева, Азербайджан. арх. Захри Хадид
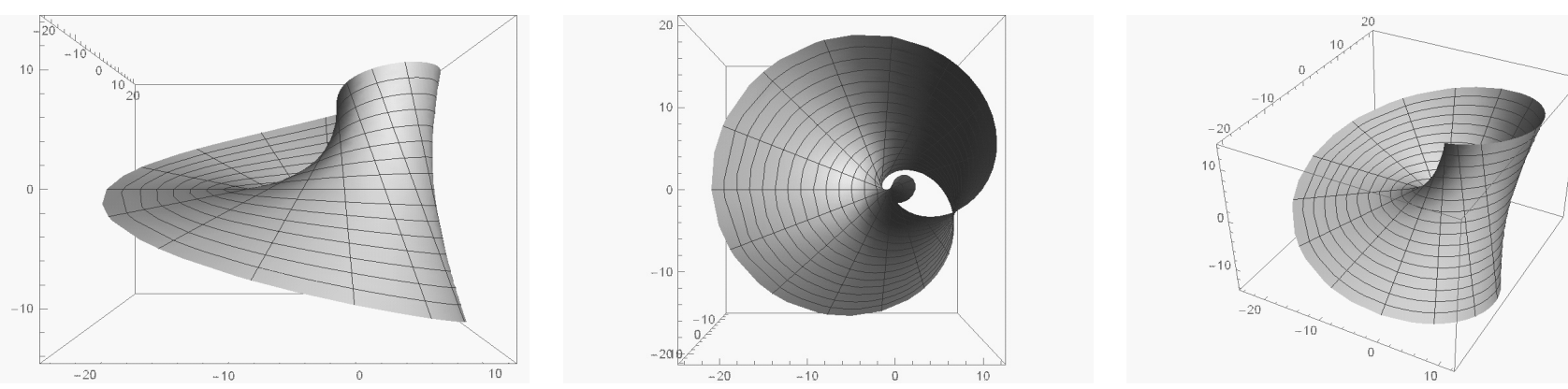

Рис. 2. Лист Мебиуса
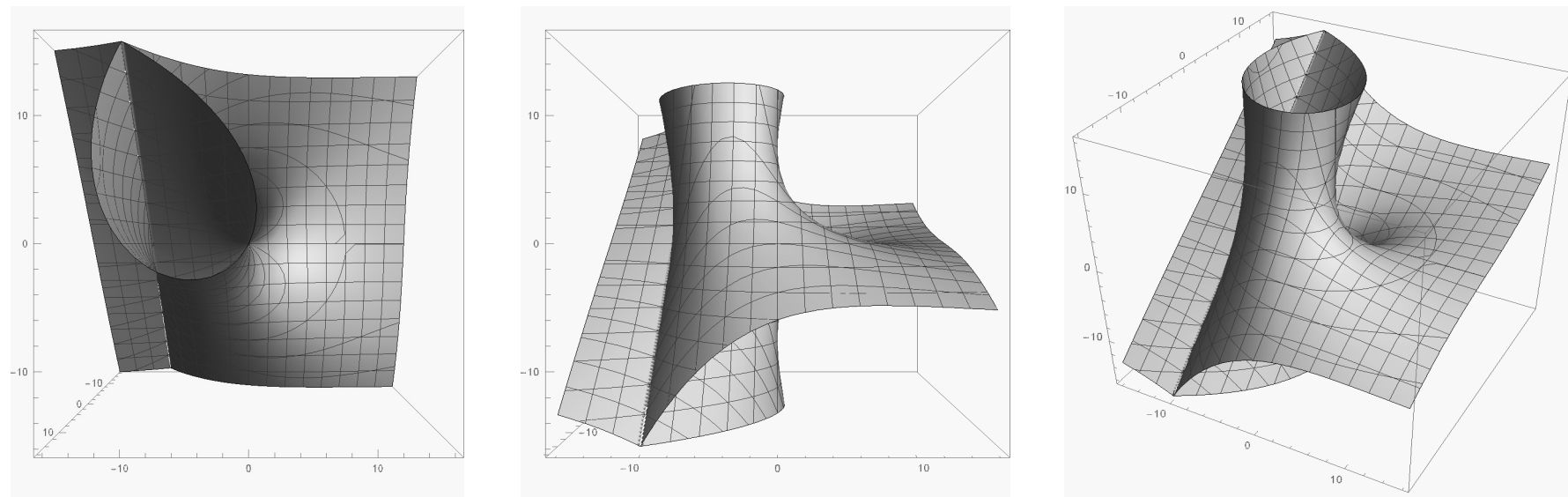

Рис. 3. Лист Мебиуса неограниченной ширины 


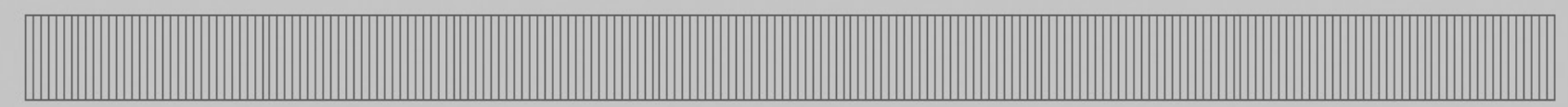

Рис. 4. Трехмерный примитив - прямоугольник

Рис. 5. Скрученный на 180 градусов лист

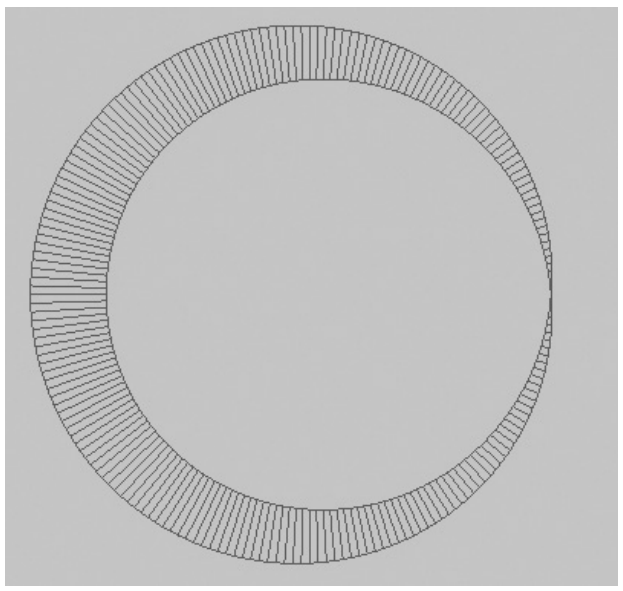

Рис. 6. Лист, свернутый в кольцо

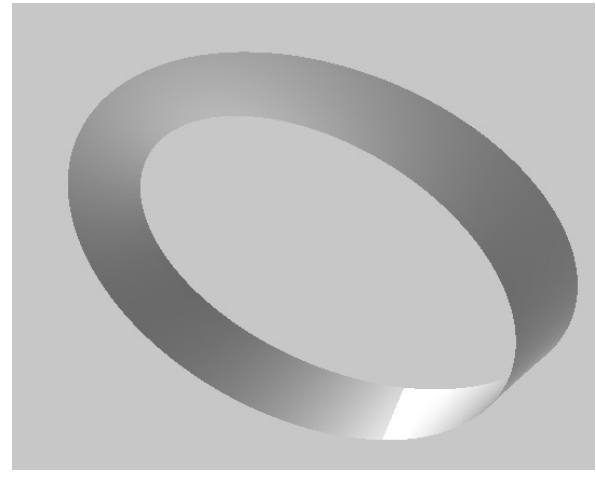

Рис. 7. Визуальное изображение Листа Мебиуса 
ной поверхности [4]. Поэтому на изображении видов присутствует линия самопересечения, которая появится и в предыдущем варианте, если в параметрических уравнениях ширину ленты сделать достаточно большой (рис. 3).

Моделирование листа Мёбиуса в программе 3dsMAX осуществляется следующим образом. Вначале создается трехмерный примитив BOX с параметрами длины, ширины и высоты - 200, 0 и 10 соответственно. Получаем длинный узкий прямоугольник. Для того, чтобы в дальнейшем его можно было изгибать, необходимо назначить 200 сегментов (максимальное значение) по наибольшей стороне (рис. 4).

На втором этапе нужно применить модификатор скручивания (Twist), установив параметр угла 180 градусов, и выбрав ось, соответствующую наибольшему габаритному размеру параллелепипеда. В результате получим скрученный на 180 градусов лист (рис. 5).

На следующей стадии преобразований применяем модификатор изгиба (Bend), установив ось изгиба, перпендикулярную полученной ленте, и параметр угла 360 градусов, в результате чего лист свернется в кольцо, и замкнется по коротким отрезкам границы (рис. 6).
После этого модели можно придать материал, и произвести рендер (т.е. получить проработанное с учетом цвета, оптических свойств выбранного материала, и освещенности визуальное изображение) (рис. 7).

\section{Выво $\triangle \mathrm{b}$}

Перспективы использования односторонней кольцевой поверхности - Листа Мебиуса достаточно широки. Инновационные формы архитектурных и дизайнерских решений [5] стало возможным воплотить с развитием технологий информационного моделирования. Информационный двойник здания будет использоваться не только на этапе проектирования и возведения, но и в процессе эксплуатации, а также утилизации сооружения. Именно по этой причине вычислительное проектирование приобретает все большую актуальность. Предварительная визуализация сложных геометрических форм, исследование восприятия проектируемых объектов с разных ракурсов, синхронизация изменений в различных частях проекта делают труд проектировщика подлинно творческим и производительным, избавляют от рутинных работ. Параметризация и создание библиотек различных поверхностей, для использования в компьютерном моделировании, весьма актуальная задача сегодняшнего этапа развития технологий проектирования.

\section{ЛИТЕРАТУРА}

1. Еремеева А.П., Наумянов Д.Э., Крылова О.В., Царева М.В. Архитектура сквозь призму геометрии. В сборнике: Дни студенческой науки. М: изд. НИУ МГСУ 2019. C. 772-774.

2. Левитин Карл «Геометрическая рапсодия», изд. Амфора,2016, 313 с.

3. Чешкова М. Инверсия листа Мёбиуса // Известия Алтайского государственного университета, 2017. № 4(96).

4. Чешкова М.А. Односторонние поверхности. Барнаул. Журнал «Известия алтайского государственного университета». 2015. № 1-2 (85) с. 164-168.

5. Кравченко Г.М. и др. Эволюция объектов параметризма. М: изд. Издательский Центр РИОР. Журнал «Строительство и архитектура» 2018. № 4 с. 44-48. 